\title{
The Impact of Patient to Nurse Ratio on Quality of Care and Patient Safety in the Medical and Surgical Wards in Malaysian Private Hospitals: A Cross-sectional Study
}

\author{
Mu’taman Jarrar ${ }^{1}$, Hamzah Abdul Rahman ${ }^{1} \&$ Abdul Shukor Shamsudin ${ }^{1}$ \\ ${ }^{1}$ College of Business, Universiti Utara Malaysia, Kedah, Malaysia \\ Correspondence: Mu'taman Jarrar, RN, MHSM, College of Business, Universiti Utara Malaysia, PO Box 36 2D \\ Sisiran, Kedah, Malaysia. Tel: 60-112-894-4581. E-mail: mutaman.jarrar@yahoo.com
}

Received: January 7, 2015 Accepted: February 7, 2015 Online Published: April 2, 2015

doi:10.5539/ass.v11n9p326 URL: http://dx.doi.org/10.5539/ass.v11n9p326

\begin{abstract}
Background and objective: Nursing shortage and inadequate hospital nursing jeopardized quality of care and patient safety. This study aims to predict the impact of patient to nurse ratio on quality of care and patient safety in medical and surgical wards in Malaysian private hospitals.

Methods: Cross-sectional data collected by questionnaire from 652 nurses working in the medical and surgical wards in 12 private hospitals participated in the study. Stratified simple random sampling performed to invite small size (less than 100 beds), medium size (100-199 beds) and large size (over than 200) hospitals' nurse to participate in the study, which allow also nurses from all shift to participate in the study from the participated hospitals.

Results: Nurses with higher ratio of patients have greater negative association on quality of care and patient safety. However, this negative association significantly associated with patient safety, whereas insignificantly associated with quality of care.

Conclusions: Staffing level inconsistently associated with quality of care and patient safety, so there is at least one intervening process factor mitigate the negative impact of nursing shortage on quality of care in Malaysian hospitals. However, nurses delivering care for 11-15 patients and nurses delivering care for more than 15 patients had significant negative impact on both quality of care and patient safety at a $p<0.05$ significance level compared with those caring for less than 5 patients.
\end{abstract}

Keywords: patient to nurse ratio, nurse adequacy, hospital size, staffing level, quality of care, patient safety

\section{Introduction}

To 'Err Is Human' report by an Institute of Medicine (IOM), stated that 98,000 deaths occurred annually as result of medical errors in United States (IOM, 2000). Adverse events occurred for $17 \%$ of all admitted patients in Australia (Wilson et al., 1995). In European countries there are inadequate hospital nursing to fulfill the rising demand in the health care facilities which in turn associated negatively with quality of care and patient safety (Hinno, Partanen, \& Vehviläinen-Julkunen, 2011). However, in Malaysia increasing demand and cost of care with lack of resources threaten the performance of Malaysian health system (Country health plan MOH, 2011). Whereas, the mismatch between patient flow and staffing level lead to increase workload which in turn threatens the performance of care (Boyer et al., 2012). Furthermore, according to the Annual Report of Ministry of Health $(\mathrm{MOH})$ Malaysia (2011) the performance of nurses working in private hospitals lower than nurses working in public hospitals $(\mathrm{MOH}, 2011)$. Thus, the main purpose of the study is to explore the effect of staffing adequacy on quality of care and patient safety in Malaysian private hospitals.

Adequate staffing is important to sustain quality of patient care (Aiken, Clarke, \& Sloane, 2002; Needleman, Buerhaus, Mattke, Stewart, \& Zelevinsky, 2002; Newhouse, Himmelfarb, \& Morlock, 2013). Study conducted in 12 European country and US found that, better working environment and low patient to nurse ratio improve quality of care, patient safety and patient satisfaction (Aiken et al., 2012). Furthermore, higher nursing ratio in the department increase their time spending with patients which in turn affect the outcome of patients care (Brooten, Youngblut, Kutcher, \& Bobo, 2004). Thus, adequate staffing level is required to improve quality of 
patient care (Newhouse et al., 2013). However, one study conducted in China found that high patient nurse ratio negatively associated with quality of care and job outcome but not associated with patient care outcome (You et al., 2013). This, finding raise the interest of investigating the impact of patient to nurse ratio in one more East Asian country like Malaysia. However, , it is difficult to identify the causal relationship between high workload and quality of patient care, and should consider patients and providers related factors which affecting the treatment process and outcome (Hillner, Smith, \& Desch, 2000). Thus, data was obtained from nurses in the medical and surgical wards of the private hospitals in Malaysia to determine the impact of staffing adequacy on quality of patient care and patient safety in order to control patient related factors affecting the outcomes. Furthermore, medical and surgical wards were chosen because they deliver multidisciplinary level of care: medical cardiology, oncology, gastroenterology, nephrology, urology, orthopedics, and ear, nose and throat treatment (Coetzee, Klopper, Ellis, \& Aiken, 2013). It can be concluded that the main purpose of the study is to investigate the impact of patient to nurse ratio on quality of care and patient safety in medical and surgical wards in Malaysian private hospitals.

\section{Method}

\subsection{Design}

A cross-sectional survey conducted at individual nurse level of analysis of nurses working in the medical and surgical wards in Malaysian private hospitals.

\subsection{Sampling}

Stratified simple random sampling performed to collect data from nurses working in the medical and surgical wards. A total 652 nurses working in the medical and surgical wards in 12 private hospitals participated in the study. The stratified random sampling offers more homogeneity within the stratum and higher heterogeneity among group of strata which produce a "mirror image of the population" (Sekaran \& Bougie, 2010). The simple random sampling of each stratum ensure each hospital and nurse have an equal chance to be chosen randomly (Sekaran \& Bougie, 2010). The inclusion criteria of hospitals are those hospitals registered in the Association of Private Hospitals of Malaysia. While the inclusion criteria of nurses are those nurses' licensed and registered by the MOH Malaysia and delivering direct inpatient care in the medical and surgical wards. According to the current nursing literature number of beds used to evaluate the hospital size to small size (less than 100 beds), medium size (100-199 beds) and large size (over than 200) hospitals (Gok \& Sezen, 2013; Lee \&Yang, 2009).

\subsection{Operationalization and Measurement}

Quality of care according to the IOM is "the degree to which health services for individuals and populations increase the likelihood of desired health outcomes and are consistent with current professional knowledge" (IOM, 2000). The outcome quality reflects the end result of interventions and treatment of the caring (Harvey, 2004). The outcome quality had two dependent variables of the study: quality of care and patient safety. Items used of measuring these variables are internationally validated (Aiken et al., 2012; Coetzee et al., 2013; Van Bogaert, Clarke, Vermeyen, Meulemans, \& Van de Heyning, 2009).

The quality of patient care was measured by asking the nurses to grade the overall quality of care in the last shift and in the last year (Van Bogaert, Meulemans, Clarke, Vermeyen, \& Van de Heyning, 2009). Furthermore, nurses were asked whether they will recommend the hospital to their friends and family if they need hospital care or as good place to work (Coetzee et al., 2013).

Patient safety in the study refers to prevent any potential harm or adverse events for hospitalized patient (Groene et al., 2010). The adverse events are unexpected patient harm or negative consequences related to patient hospitalization other than his disease process (Weingart et al., 2011), and this also called hospital acquired conditions. For instance, adverse events could be hospital acquired infection which also called nosocomial infection. In addition, pressure ulcer, patient fall, medication errors and readmission also considered as adverse events (Weingart et al., 2011; Welton, 2008). According to the current nursing literature the common events in the medical and surgical wards are: nosocomial infection, patient falls, medication error and patient and family complaints (Laschinger \& Leiter, 2006; Van Bogaert et al., 2014). One item from the Agency for Healthcare Research and Quality survey of patient safety used to rate the overall patient safety in their unit using five point Likert scale (Aiken et al., 2012; Coetzee et al., 2013; You et al., 2013). The second measure of patient safety of this study is the adverse events which include: nosocomial infection, pressure ulcer, patient fall, medication errors, readmission and patient and family complaints (Laschinger \& Leiter, 2006; Van Bogaert et al., 2014; Weingart et al., 2011). Thus, nurses were asked to report their degree of agreement of the overall rating of patient safety and their rating of the frequency of the adverse events. 
The patient-nurse ratio was calculated by asking the nurses to indicate how many patients directly assigned under his care on last shift (Aiken et al., 2012; Coetzee et al., 2013; You et al., 2013), and the number of patient was chosen from four categories: less than 5 patients, 5-10 patients, 11-15 patients and over than 15 patients. The lower ratio indicate more favorable nursing staffing (Aiken et al., 2012).

Back-To-Back translation is performed to make sure that questionnaire free of mistakes, wrong wording or changing in the meaning. Furthermore, questionnaire translation to the local language (Bahasa Melayu) by local expert and then back translation by another expert to ensure the conceptual and vocabulary equivalence of the questionnaire items (Sekaran, 2003).

\subsection{Data Analysis}

Multiple regression analysis performed using the SPSS software version 21.0 to investigate the impact of patient to nurse ratio on quality of care and patient safety at significance level $p<0.05$. A pilot study conducted to test the internal consistency in order to check the instrument adequacy and the soundness of questionnaire by measuring Cronbach's alpha coefficient. The Cronbach's alpha coefficient of the quality of care and patient safety was 0.75 and 0.85 respectively, which were above the recommended level of Cronbach's alpha coefficient 0.70 (Pallant, 2011; Sekaran \& Bougie, 2010).

\section{Results}

A total 1055 of registered nurses working on the medical and surgical wards in the 12 participating hospitals were invited to participate in the study. A total 807 questionnaires were returned back, so $76.4 \%$ is the total response rate of participant nurses in the study. However, 155 respondents were excluded from the study because they not met the inclusion criteria (nurses not working in the medical and surgical wards), and others have fixed response to of all questionnaire items, or left it blank. Thus, the usable respondents remained are 652 to proceed in data analysis, representing $61.8 \%$ response rate.

The demographical characteristics as shown indicated that $99.0 \%$ of nurses participated in the study were Malaysian, and $97.6 \%$ of nurses were female. Furthermore, the Malay nurses have the higher proportion of participants in the study $60.0 \%$, while $21.6 \%$ Chinese, $14.2 \%$ Indian and $2.2 \%$ others (the others included Thais and Filipinos nurses). Most of the nurses were between the age 25 and 30 years $43.7 \%$. The majority of respondents hold diploma $84.6 \%$ while $10.3 \%$ had bachelor degree in nursing and $5.1 \%$ other (the others included nurses had associate degree of nursing or higher education). In term of job title the majority of nurses were staff nurses $90.4 \%$, while only $6.2 \%$ are assistant nurses and $3.4 \%$ nurses others (the others included the community nurses, in-charge nurse) and all of them delivering direct inpatient care. The status of employment of nurses in the study were $98.1 \%$ full time while $1.9 \%$ were part time nurses. The majority of nurses were working in large size hospitals $72.2 \%$, while $16.5 \%$ working in medium size hospitals and $11.3 \%$ working in small size hospitals.

The patient-nurse ratio show that $37.0 \%$ of nurses working in Malaysian private hospitals provided care for more than 15 patients under their care in one shift, whereas $24.0 \%$ caring for $11-15$ patients, $31.2 \%$ caring for $5-10$ patients and $7.7 \%$ caring for less than 5 patients, under their assignment in the last shift they worked. Furthermore, $23.3 \%$ nurses participated in the study working in the medical ward, $26.1 \%$ in the surgical ward, $7.4 \%$ in the general ward, $31.9 \%$ in the multidisciplinary ward and $11.3 \%$ others (the others refer to endoscopy, oncology, cardiology and cardiothoracic wards that nurses provide direct inpatient care), and all of these wards deliver a multidisciplinary level of care.

The patient-nurse ratio construct included four categories, thus $\mathrm{j}-1$ dummy variables to capture all information for each category compared with the reference group (Cohen, Cohen, West, \& Aiken, 2003; Hardy, 1993; West, Aiken, \& Krull, 1996). The reference group should be the group which is expected to score highest or lowest in relation with the dependent variable (Cohen et al., 2003; Hardy, 1993; West et al., 1996). Moreover, the reference group, should be well defined in order to clearly interpret the regression result, and should not be "others category" (Cohen et al., 2003; Hardy, 1993; West et al., 1996). Thus, the reference group in the study was nurses caring with less than 5 patients.

Multiple regression analysis of the effect of patient to nurse ratio on the outcomes of care were explored two regression models. The first model explored the effect of patient to nurse ratio on quality of care, whereas the second regression model was explored the effect of patient to nurse ratio on patient safety.

\subsection{Model 1: Patient to Nurse Ratio and Quality of Care}

Patient to nurse ratio dimension included three dummy variables. Table 1 provided the result of multiple regression analysis of its impact on quality of care in order to test the hypothesis: 
The alternative hypothesis H1: Patient to nurse ratio is associated with quality of care.

The null hypothesis $\mathrm{H1}_{0}$ : Patient to nurse ratio is not associated with quality of care.

Table 1. Regression result of patient to nurse ratio on quality of care

\begin{tabular}{cccccc}
\hline Variables & $\begin{array}{c}\text { Unstandardized } \\
\text { Coefficients B }\end{array}$ & Std. Error & $\begin{array}{c}\text { Standardized } \\
\text { Coefficients } \beta\end{array}$ & $\mathrm{t}$ & Sig. \\
\hline $\begin{array}{c}\text { (Constant) } \\
\text { Less than 5 patients (R) }\end{array}$ & 3.90 & 0.08 & & 46.46 & 0.00 \\
5-10 patients & -0.12 & 0.09 & -0.10 & -1.27 & 0.20 \\
11-15 patients & $-0.21^{*}$ & 0.10 & -0.16 & -2.13 & 0.03 \\
over 15 patients & $-0.22^{*}$ & 0.09 & -0.19 & -2.38 & 0.02 \\
\hline $\mathrm{R}^{2}$ & 0.01 & & & & \\
F value & 2.61 & & & & \\
Significance of $\mathrm{F}$ value & 0.05 & & &
\end{tabular}

The result of the regression analysis as shown in Table 1 revealed that $\mathrm{F}=2.61$ and $\mathrm{P}$ value $=0.05$ indicated that the study failed to reject the null hypothesis $\mathrm{H}_{0}$. So, the relationship of patient nurse ratio on quality of care is not significant. The $\mathrm{R}^{2}$ indicated that nurse to patient ratio variable predict 0.01 of variances in quality of care and not significant at level $\mathrm{p}<0.05$. However, the unstandardized coefficient of the three dummy variables indicated that increasing patient to nurse ratio is negatively associated with quality of care, and by increasing number of patients assigned under each nurse this negative impact is increasing $-0.12,-0.21$, and -0.22 respectively. Furthermore, nurses delivering care for $11-15$ patients $(B=-0.21, t=-2.13, p=0.03)$ and over than 15 patients $(\mathrm{B}=-0.22, \mathrm{t}=-2.38, \mathrm{p}=0.02)$ have significant negative impact on quality of care at a $\mathrm{p}<0.05$ significance level compared with those caring with less than 5 patients.

\subsection{Model 1: Patient to Nurse Ratio and Patient Safety}

Table 2 provided the result of multiple regression analysis of its impact on patient safety in order to test the hypothesis:

The alternative hypothesis H2: Patient to nurse ratio is associated with patient safety.

The null hypothesis $\mathrm{H}_{2}$ : Patient to nurse ratio is not associated with patient safety.

Table 2. Regression result of patient to nurse ratio on patient safety

\begin{tabular}{lccccc}
\hline Variables & $\begin{array}{c}\text { Unstandardized } \\
\text { Coefficients B }\end{array}$ & Std. Error & $\begin{array}{c}\text { Standardized } \\
\text { Coefficients } \beta\end{array}$ & $\mathrm{t}$ & Sig. \\
\hline (Constant) & 3.80 & 0.09 & & 43.46 & 0.00 \\
Less than 5 patients (R) & & & & & \\
5-10 patients & -0.18 & 0.10 & -0.14 & -1.81 & 0.07 \\
$11-15$ patients & $-0.25^{*}$ & 0.10 & -0.18 & -2.44 & 0.02 \\
over 15 patients & $-0.26^{* *}$ & 0.10 & -0.21 & -2.66 & 0.01 \\
\hline $\mathrm{R}^{2}$ & 0.01 & & & & \\
F value & $2.73^{*}$ & & & & \\
Significance of F value & 0.04 & & &
\end{tabular}

The result of the regression analysis as shown in Table 2 revealed that $\mathrm{F}=2.73$ and $\mathrm{P}$ value $=0.04$ indicated that the study reject the null hypothesis $\mathrm{H} 2{ }_{0}$. So, the relationship of patient nurse ratio on patient safety is significant. The R2 indicate that nurse to patient ratio variable predict 0.01 of variances in patient safety and significant at level $p<0.05$. Moreover, the unstandardized coefficient of the three dummy variables indicated that increasing patient to nurse ratio is negatively associated with patient safety, and by increasing number of patients assigned under each nurse this negative impact is increasing $-0.18,-0.25$, and -0.26 respectively. Furthermore, nurses delivering care for $11-15$ patients $(\mathrm{B}=-0.25, \mathrm{t}=-2.44, \mathrm{p}=0.02)$ and over than 15 patients $(\mathrm{B}=-0.26, \mathrm{t}=-2.66, \mathrm{p}=0.01)$ 
have significant negative impact on patient safety at a $\mathrm{p}<0.05$ significance level compared with those caring with less than 5 patients among nurses working in medical and surgical wards in Malaysian private hospitals. Thus, it can be concluded that the hypothesis H2 supported, while H1 not supported.

\section{Discussion}

Regression analysis of the effect of patient to nurse ratio as shown in both Table 1 and Table 2 indicated insignificant impact on quality of care and significant negative impact on patient safety at $p<0.05$ significance level, respectively. These findings inconsistent with the previous studies (Aiken et al., 2012; Boyer et al., 2012; Brooten et al., 2004; Coetzee et al., 2013). Boyer et al. (2012) found that mismatch between patient flow and staffing level lead to increase workload which in turn lowers the outcomes of care. In addition, one study conducted in 12 European country and in US found that, low patient to nurse ratio enhance quality of care, patient safety and patient satisfaction (Aiken et al., 2012). Thus, adequate staffing is required to improve quality of patient care (Aiken et al., 2002; Needleman et al., 2002; Newhouse et al., 2013). However, a study conducted in China found that high patient to nurse ratio negatively associated with quality of care and job outcome but not associated with patient care outcome (You et al., 2013). Furthermore, other studies found inconsistent relationship between patient to nurse ratio and the outcomes (Needleman et al., 2002). These inconsistent findings supported this study finding and show the importance to investigate an intervening variable with opposite sign suppressing the relationship of patient to nurse ratio on quality of care.

High patient to nurse ratio indicate unfavorable nursing staffing affecting the outcomes (Aiken et al., 2012), and this finding is consistent with the present study. The unstandardized beta coefficient reported in Table 1 revealed that increasing patient to nurse ratio is negatively associated with quality of care, and by increasing number of patients assigned under each nurse this negative impact is increasing $-0.12,-0.21$, and -0.22 respectively. Similarly, it is negatively associated with patient safety, and by increasing number of patients assigned under each nurse this negative impact is increasing $-0.18,-0.25$, and -0.26 respectively. Furthermore, nurses delivering care for 11-15 patients and nurses delivering care for over than 15 patients have significant negative impact on both quality of care and patient safety at a $<<0.05$ significance level compared with those caring with less than 5 patients. Thus, assigning more than 10 patients under one nurse is threatening quality of care and patient safety in the medical and surgical wards in Malaysian private hospitals. However, it is difficult to identify the causal relationship between high hospital volume and quality of patient care, and should consider patients and providers related factors, this affect the treatment process and outcome (Hillner et al., 2000). The present study controlled the variances of the patient related factors affecting the outcomes. Future research suggested will be control the process factors affecting the outcomes of care. For instance, nursing task oriented and patient and family involvement in care mitigate the negative impact of nursing shortage (You et al., 2013). Moreover, low patient to nurse ratio in the ward increases the time spent with patients which in turn affect the outcome of patients care (Brooten et al., 2004). Thus, the strong argument for future research is to explore the effect of patient centeredness on the relationship of nursing shortage on quality of care and patient safety in Malaysian private hospitals.

\section{Conclusion}

Inadequate staffing, nursing shortage and high patient to nurse ratio can jeopardize quality of care and patient safety in the medical and surgical wards in Malaysian private hospitals. However, there is at least one process factor with positive sign mitigate this negative impact of nursing shortage on quality of care in Malaysian private hospitals. The $\mathrm{R}^{2}$ of the regression result indicated that only $1 \%$ of variances of quality and patient safety explained by the nurse to patient ratio. Thus, the authors proposed focus on process intervening factors affecting the outcomes of care as a remedy of improving quality of care and patient safety.

\section{Acknowledgments}

Grant for the research is provided by Universiti Utara Malaysia. The authors acknowledge Prof. Dr. Mohammad Alghoul from the National University of Malaysia and Dr. Liana Orsolini-Hain PhD, RN and Care Delivery and Advanced Practice Systems Consultant in Bon Secours Health System, Inc (BSHSI) for their guidance and support for this work.

\section{References}

Aiken, L. H., Clarke, S., \& Sloane, D. (2002). Hospital nurse staffing and patient mortality, nurse burnout, and job dissatisfaction. Jama, 288(16), 1987-1993. Retrieved from http://jama.jamanetwork.com/article. aspx? articleid $=195438$

Aiken, L. H., Sermeus, W., Van den Heede, K., Sloane, D. M., Busse, R., Mckee, M., ... Smith, H. L. (2012). 
Patient safety, satisfaction, and quality of hospital care : cross sectional surveys of nurses and patients in 12 countries in Europe and the United States. British Medical Journal, 344(e1717), 1-14. http://dx.doi.org/10.1136/bmj.e1717

Boyer, S., Protopopescu, C., Marcellin, F., Carrieri, M. P., Koulla-shiro, S., Moatti, J., ... Group, S. (2012). Performance of HIV care decentralization from the patient's perspective : health-related quality of life and perceived quality of services in Cameroon. Health Policy and Planning, 27(June 2011), 301-315. http://dx.doi.org/10.1093/heapol/czr039

Brooten, D., Youngblut, J. M., Kutcher, J., \& Bobo, C. (2004). Quality and the nursing workforce: APNs, patient outcomes and health care costs. Nursing outlook, 52(1), 45-52. http://dx.doi.org/10.1016/j.outlook. 2003.10.009

Coetzee, S. K., Klopper, H. C., Ellis, S. M., \& Aiken, L. H. (2013). A tale of two systems--nurses practice environment, well being, perceived quality of care and patient safety in private and public hospitals in South Africa: a questionnaire survey. International journal of nursing studies, 50(2), 162-73. http://dx.doi.org/10.1016/j.ijnurstu.2012.11.002

Cohen, J., Cohen, P., West, S., \& Aiken, L. (2003). Applied multiple regression/correlation analysis for the behavioral sciences. Lawrence Erlbaum Associates. Retrieved from http://books.google.com/books?hl= en $\& 1 \mathrm{r}=\& \mathrm{id}=$ gkalyqTMXNEC\&oi=fnd\&pg=PP1\&dq=Applied + multiple + regression/+correlation + analysis + for + the+behavioral + sciences \& ots $=$ tQCV02 mbch\&sig=kbtLMNFzquJwmyNgVxNV-Owh07s

Country health plan MOH. (2011). Country health plan 10 th Malaysia plan 2011-2015 (p. 82). Retrieved from http://www.moh.gov.my/images/gallery/Report/Country_health.pdf

Gok, M. S., \& Sezen, B. (2013). Analyzing the ambiguous relationship between efficiency, quality and patient satisfaction in healthcare services: the case of public hospitals in Turkey. Health policy (Amsterdam, Netherlands), 111(3), 290-300. http://dx.doi.org/10.1016/j.healthpol.2013.05.010

Groene, O., Klazinga, N., Wagner, C., Arah, O. a, Thompson, A., Bruneau, C., \& Suñol, R. (2010). Investigating organizational quality improvement systems, patient empowerment, organizational culture, professional involvement and the quality of care in European hospitals: the "Deepening our Understanding of Quality Improvement in Europe (DUQuE)" proje. BMC health services research, 10, 281. http://dx.doi.org/10.1186/1472-6963-10-281

Hardy, M. (1993). Regression with dummy variables (1st ed.). SAGE Publications Ltd. Retrieved from http://books.google.com/books?hl=en\&lr=\&id=EzLffJlYISEC\&oi=fnd\&pg=PR5\&dq=Regression+with+D ummy+variable\&ots $=$ R81XjXqfSx \&sig=lnNBq6o1hwewjSygDJeQDJSyJTw

Harvey, R. (2004). Using clinical performance information to improve the quality of care in a specialist NHS trust. Journal of nursing management, 12(6), 427-435. http://dx.doi.org/10.1111/j.1365-2834.2004.00513.x

Hillner, B. E., Smith, T. J., \& Desch, C. E. (2000). Hospital and physician volume or specialization and outcomes in cancer treatment: importance in quality of cancer care. Journal of clinical oncology : official journal of the American Society of Clinical Oncology, 18(11), 2327-2340. Retrieved from http://www.ncbi.nlm.nih.gov/pubmed/10829054

Hinno, S., Partanen, P., \& Vehviläinen-Julkunen, K. (2011). Hospital nurses’ work environment, quality of care provided and career plans. International nursing review, 58(2), 255-262. http://dx.doi.org/10.1111/j.14667657.2010.00851.x

IOM. (2000). To Err Is Human: Bulding a Safer Health System. National Academy Press, Washington DC.

Laschinger, H., \& Leiter, M. (2006). The impact of nursing work environments on patients safety outcomes. The mediating role of burnout/engagement. Journal of Nursing Administration, 36, 259-267.

Lee, K. H., Yang, S. B., \& C. M. (2009). The association between hospital ownership and technical efficiency in a managed care environment. Journal of Medical Systems, 33, 307-315.

MOH. (2011). Annual Report (p. 351). Retrieved from http://www.moh.gov.my/images/gallery/publications/ $\mathrm{md} / \mathrm{ar} / 2011$ en.pdf

Needleman, J., Buerhaus, P., Mattke, S., Stewart, M., \& Zelevinsky, K. (2002). Nurse-staffing levels and the quality of care in hospitals. The New England journal of medicine, 346(22), 1715-1722. http://dx.doi.org/ 10.1056/NEJMsa012247

Newhouse, R. P., Himmelfarb, C. D., \& Morlock, L. (2013). A phased cluster-randomized trial of rural hospitals 
testing a quality collaborative to improve heart failure care: organizational context matters. Medical Care, 51(5), 396-403.

Pallant, J. (2011). SPSS Survival Manual (4th ed., p. 359). British library. Retrieved from http://scholar.google.com/scholar?hl=en\&btnG=Search\&q=intitle:SPSS+Survival+Manual\#7

Sekaran, U. (2003). RESEARCH METHODS FOR BUSINESS (4th ed., p. 466). John Wiley \& Sons Ltd.

Sekaran, U., \& Bougie, R. (2010). Research Methodology for Business (5th ed., p. 468). John Wiley \& Sons Ltd.

Van Bogaert, P., Clarke, S., Vermeyen, K., Meulemans, H., \& Van de Heyning, P. (2009). Practice environments and their associations with nurse-reported outcomes in Belgian hospitals: development and preliminary validation of a Dutch adaptation of the Revised Nursing Work Index. International journal of nursing studies, 46(1), 54-64. http://dx.doi.org/10.1016/j.ijnurstu.2008.07.009

Van Bogaert, P., Meulemans, H., Clarke, S., Vermeyen, K., \& Van de Heyning, P. (2009). Hospital nurse practice environment, burnout, job outcomes and quality of care: test of a structural equation model. Journal of Advanced Nursing, 65(10), 2175-2185. http://dx.doi.org/10.1111/j.1365-2648.2009.05082.x

Van Bogaert, P., Timmermans, O., Weeks, S. M., van Heusden, D., Wouters, K., \& Franck, E. (2014). Nursing unit teams matter: Impact of unit-level nurse practice environment, nurse work characteristics, and burnout on nurse reported job outcomes, and quality of care, and patient adverse events-A cross-sectional survey. International journal of nursing studies, 51(8), 1123-1134. http://dx.doi.org/10.1016/j.ijnurstu.2013.12.009

Weingart, S. N., Zhu, J., Chiappetta, L., Stuver, S. O., Schneider, E. C., Epstein, A. M., ... Weissman, J. S. (2011). Hospitalized patients' participation and its impact on quality of care and patient safety. International journal for quality in health care : journal of the International Society for Quality in Health Care / ISQua, 23(3), 269-277. http://dx.doi.org/10.1093/intqhc/mzr002

Welton, J. M. (2008). Implications of Medicare reimbursement changes related to inpatient nursing care quality. The Journal of nursing administration, 38(7-8), 325-330. http://dx.doi.org/10.1097/01.NNA.0000323944. 89640.c0

West, S. G., Aiken, L. S., \& Krull, J. L. (1996). Experimental Personality Designs: Analyzing Categorical by Continuous Variable Interactions. Journal of Personality, 64(1).

Wilson, R., Runciman, W., Gibberd, R., Harrison, B., Newby, L., \& Hamilton, J. (1995). The quality in Australian health care study. Med J Aust, 163, 458-471.

You, L., Aiken, L. H., Sloane, D. M., Liu, K., He, G., Hu, Y., ... Sermeus, W. (2013). Hospital nursing, care quality, and patient satisfaction: cross-sectional surveys of nurses and patients in hospitals in China and Europe. International journal of nursing studies, 50(2), 154-161. http://dx.doi.org/10.1016/j.ijnurstu. 2012.05 .003

\section{Copyrights}

Copyright for this article is retained by the author(s), with first publication rights granted to the journal.

This is an open-access article distributed under the terms and conditions of the Creative Commons Attribution license (http://creativecommons.org/licenses/by/3.0/). 